\title{
Synchronous use of FPA-based infrared thermography and fast ellipsometry for high-sensitive investigation of the adsorption-desorption processes rapidly progressing on solid surfaces
}

\author{
by B. G. Vainer, A. A. Guzev, K. P. Mogilnikov, S. I. Romanov, V. A. Shvets
}

Rzhanov Institute of Semiconductor Physics SB RAS, Novosibirsk State University; 13 Lavrentyev av., Novosibirsk, 630090, Russia, BGV@isp.nsc.ru

\section{Introduction}

There are no satisfactory methods enabling high-sensitive laboratory monitoring and scientific investigation of the initial stages of the adsorption processes progressing on solid surfaces as soon as these surfaces are attacked by gas molecules. The same may be said about desorption. Nevertheless, an assessment of the magnitude of thermal effect arisen owing to adsorption heat release shows that FPA-based infrared thermography may be sensitive to hundredth parts of a monolayer adsorbed on the surface [1, 2].

We realized the experimental setup combining an FPA-based infrared camera and fast laser ellipsometer within a single system meant for the above-mentioned measurements. The description of its design was first briefly given in [3]. In the present contribution, we describe in details some special features of this technique and exhibit interesting results obtained during investigation of different samples: solid films, nanochannel membranes, porous silicon, etc.

\section{Measurement system}

Taking into account the objectives of the present work, it should be noted that an experimental sample cannot be precisely measured, even in laboratory conditions, if it is subjected to open atmosphere because a set of extraneous external factors, which are capable to distort information about investigated objects, exist. As far as infrared thermography is concerned, these factors may be as follows.

a) Non-uniform air temperature distribution in the laboratory room resulted from draughts, the presence of personnel near a measurement unit, air warming from measuring apparatus, etc. give rise to uncontrollable heating or cooling of a sample. An infrared camera acquiring hundreds frames per second with the temperature sensitivity of the order of $10^{-2}{ }^{\circ} \mathrm{C}$ interprets these temperature variations as a wanted signal that is inadmissibly under the conditions of the problem in question.

b) None of experimental samples may be considered as a black body. Consequently, the experimental sample surface always reflects a part of incident radiation. Infrared camera cannot separate intrinsic emission from reflexes. It requires samples shielding from the environment.

c) Cutaneous perspiration in humans results in skin water evaporation. This vapor may be absorbed by a surface of the experimental sample that may increase the surface temperature and exert some effect on the measurement data.

We created a combined measurement system which is fully guarded against all the above-mentioned artefacts.

A block diagram of the system is shown in figure 1. An exterior view of the main part of this system is shown in figure 2. Figure 3 represents the interior of a vacuum-sealed chamber equipped with the window transparent to infrared radiation and two windows transparent to laser radiation. The semiconductor sample is clearly seen deep in the box in the centre of the photograph.

\section{Experimental results}

Testing the created measurement complex was realized with the use of several types of experimental samples. Among them were: single-crystal silicon with the oxidized surface, microchannel silicon structures with a different spacing between channels (from $4 \mu \mathrm{m}$ to $20 \mu \mathrm{m}$ ), nanoporous silicon, titanic foil covered with anodic oxide, recording tape, etc. Water vapors of different concentrations served as typical adsorbate in these tests.

Figure 4 represents the fast alteration dynamics of ellipsometric angle $\Delta$ observed immediately after the hermetic container with experimental sample opened inside a vacuum-sealed chamber. Curve 1 demonstrates the process of molecules desorption from the surface of thermally oxidized silicon the initial stage after opening. During 2 seconds after opening, we can see a monotonic increase of the $\Delta$ value for $0.04^{\circ}$. It corresponds to the removal of the surface water layer of about 0.5-angstrom thickness, in other words, several tenths parts of a monolayer. Curve 2 in figure 4 does not demonstrate such an effect because it was measured when the adsorbate was already removed.

The results obtained by infrared camera are shown in figure 5. In this experiment, initially, the air was evacuated from the vacuum-sealed chamber up to the residual pressure of $0.1 \mathrm{~atm}$ which was kept at this level during 15 minutes. 


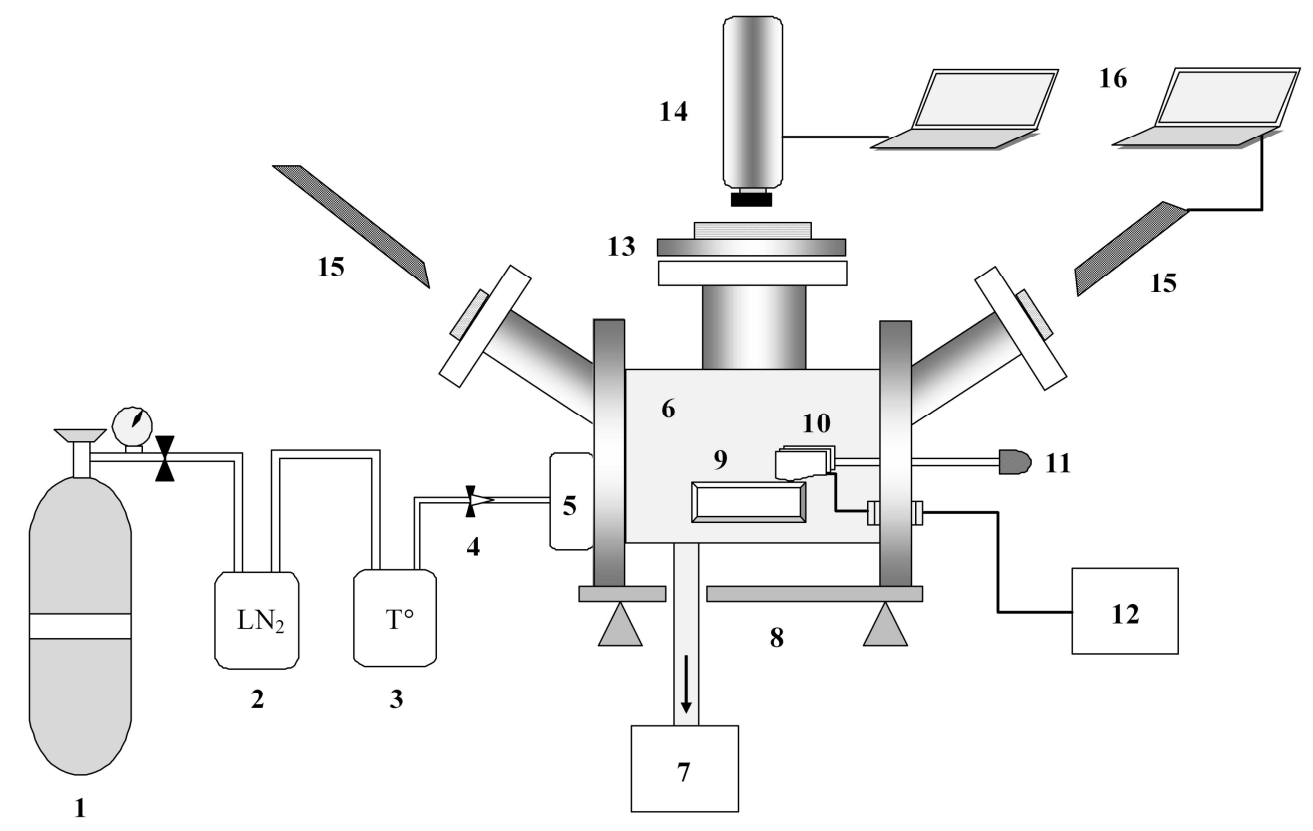

Fig. 1. Skeleton diagram of the thermographic and ellipsometric integrated measurement complex destined for a precise study of adsorption and desorption dynamic processes progressing in the course of gas molecules and solid adsorbent surfaces interaction.

1 - gas cylinder with carrier gas (argon, nitrogen, oxygen and so on), 2 - first (passive) heat exchanger (dehumidifier), 3 - second (thermocontrolled) heat exchanger filled with adsorbate in liquid or solid phase; it serves to add a preset value of the adsorbate to the mixture, 4 - inlet valve, 5-third (adapting) heat exchanger, 6 - vacuum-sealed chamber with transparent windows, 7 - vacuum line, 8 - adjusting gear, 9-leakproof container with experimental sample, 10 - electromechanical system meant for operation with the container shutter, 11 - "through-the-wall" manipulator, 12 - electronic control unit, 13 - loading channel and output window for thermographic control, 14 - infrared camera, 15 - ellipsometric arms, input and output ellipsometric windows, 16 - computer system for acquisition and processing of thermographic and ellipsometric data.

Then the adsorption pump brought the pressure to the level of the order of $10^{-3}$ atm. Figure 6 quantitatively demonstrates the effect represented in figure 5.

An example of synchronous measurement of the surface reconstructions initiated by water desorption from a thin hanging microchannel silicon membrane (figure 7) is shown in figure 8. Figure 9 demonstrates the synchronism in the experiment with porous silicon as an experimental sample.

\section{Conclusions}

A new high-sensitive and fast method of experimental investigation of interactions of gaseous adsorbate molecules and solid surfaces over a wide range of adsorbate concentrations is devised and realized. Using the created measurement system combining two methods - thermal imaging and ellipsometry - the adsorption and desorption processes are investigated in a pilot manner.

The described new approach to the quantitative investigation of physicochemical processes associated with adsorption and desorption of vapor molecules on solid surfaces consisting of synchronous recording of the data acquired by means of FPA-based infrared thermography and fast ellipsometry is endowed with great prospects.

\section{Acknowledgements}

This work was supported by the Russian Foundation for Basic Research, grant No. 11-03-00900-a and partially by the Siberian Branch of the Russian Academy of Sciences (Integrative Project No. 40-B-2012).

\section{REFERENCES}

[1] Vainer B. G. "Quantitative characterization of vapour adsorption on solid surfaces and estimation of emissivity of solids using narrow-band short-wave infrared thermography". QIRT Journal, vol. 5, pp. 175-193, 2008. 
[2] Vainer B. G. "Focal plane array based infrared thermography in fine physical experiment". J. Phys. D: Appl. Phys., vol. 41, pp. 065102, 2008.

[3] Vainer B. G., Guzev A. A., Mogilnikov K. P., Shvets V. A. "Computerized infrared thermography and ellipsometry-based instrumentation facility intended for the studies of rapid dynamics of starting stages of adsorption and desorption of gases on solid surfaces at wide range of adsorbate concentrations". - In: Education and Science: State-of-the-Art and Prospect of Development// Intern. Scientific-Practical Conf., February 28, 2013. Proceedings. Part 8. Tambov: TROO "Business-Science-Society", pp. 38-40, 2013.

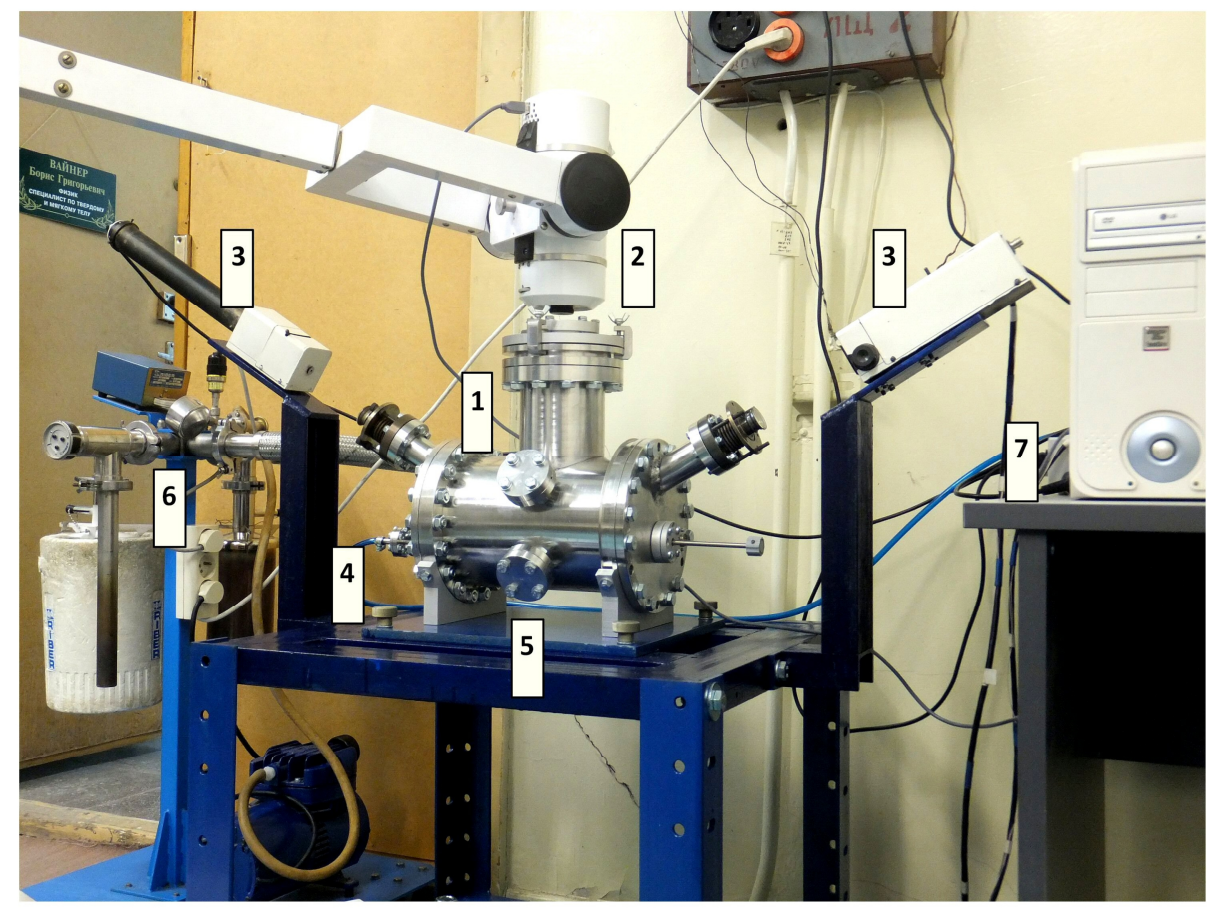

Fig. 2. Exterior view of the research facilities enabling synchronous thermographic and ellipsometric measurements of the initial stages of adsorption and desorption processes developing on solid surfaces.

1 - vacuum-sealed chamber with three optical windows, 2 - infrared camera, 3 - ellipsometer, 4 - gas (adsorbate) feed system, 5 - adjusting support, 6 - vacuum system, 7 - computer system for acquisition and processing of experimental data from ellipsometer and infrared camera.

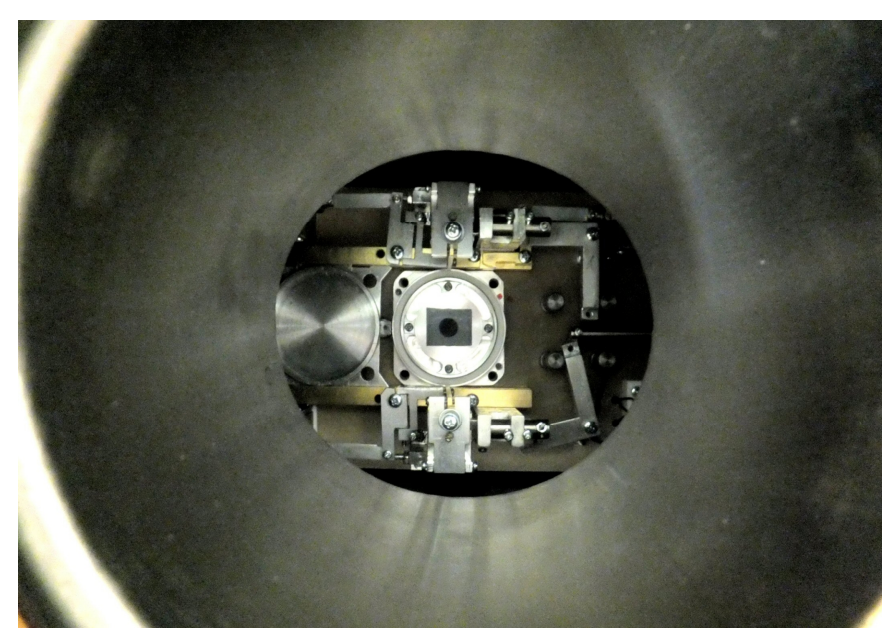

Fig. 3. The interior of a vacuum-sealed chamber. 


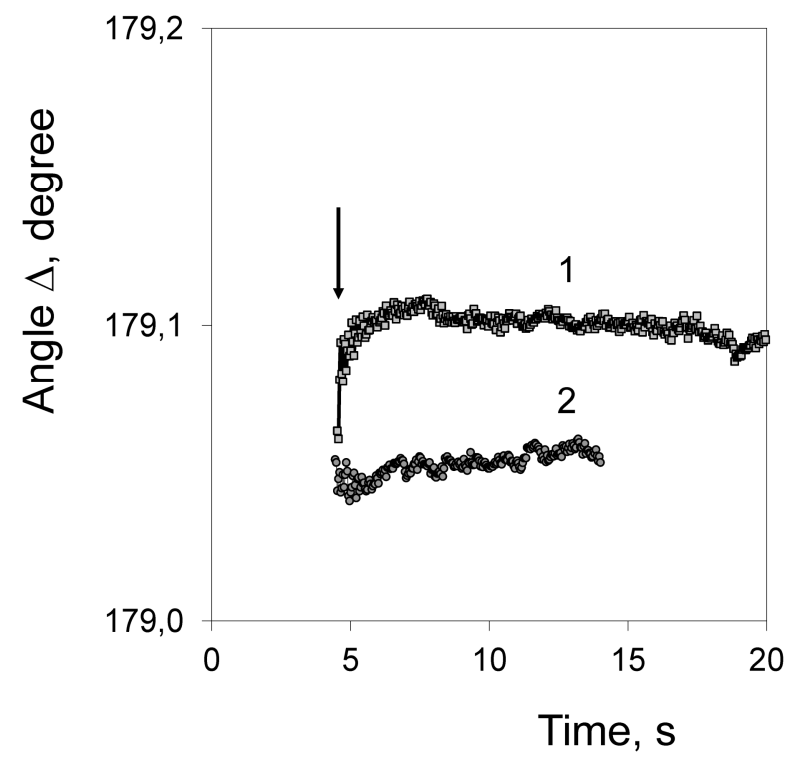

Fig. 4. The dynamics of fast alteration (indicated by arrow) of ellipsometric angle $\Delta$ immediately after the container with the experimental sample opened. 1 - after first opening, 2 - after second opening.

Curve 1 demonstrates the process of desorption of molecules from the surface of thermally oxidized silicon (oxide film thickness $525 \mathrm{~nm}$ ). Initially, the container was filled with atmospheric air under the pressure of 70 torr, and the vacuum-sealed chamber was filled with the atmospheric air under the pressure of $10^{-1}$ torr.

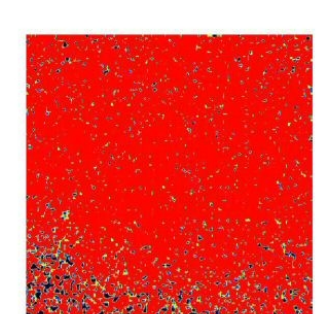

$\mathrm{n}=346$

0.175

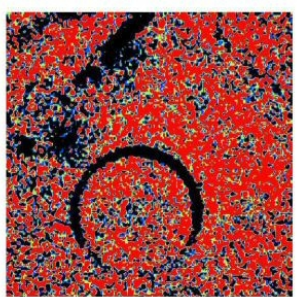

$\mathrm{n}=366$

3.703

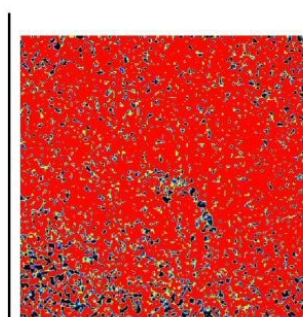

$\mathrm{n}=350$

0.877

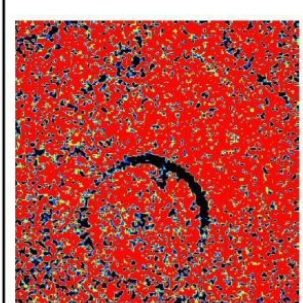

$\mathrm{n}=352$ 1.228

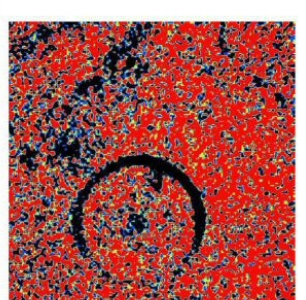

$\mathrm{n}=354$

1.578

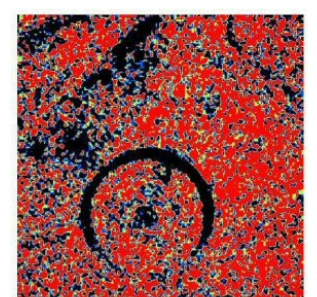

$\mathrm{n}=356$ 1.939

Fig. 5. A selection of thermograms taken from the set of sequential thermograms measured in a microchannel silicon structure (spacing: $20 \mu \mathrm{m} \times 20 \mu \mathrm{m}$ ) after the air pressure in a vacuum-sealed chamber was quickly changed from $10^{-1}$ to $10^{-3} \mathrm{~atm}$. The thermograms are obtained in the regime of subtraction of the initial reference frame.

Thermograms legends: upper line - sequence number of a frame in the continuous thermal movie, lower line - the time (in seconds) starting from the short period of pressure change. The last thermogram (the beginning of the process) in the figure is presented on a comparative purpose and for the purpose to be sure that the recording was started when all transient thermal processes associated with desorption of molecules from the surrounding constructive elements of the container are completed. 


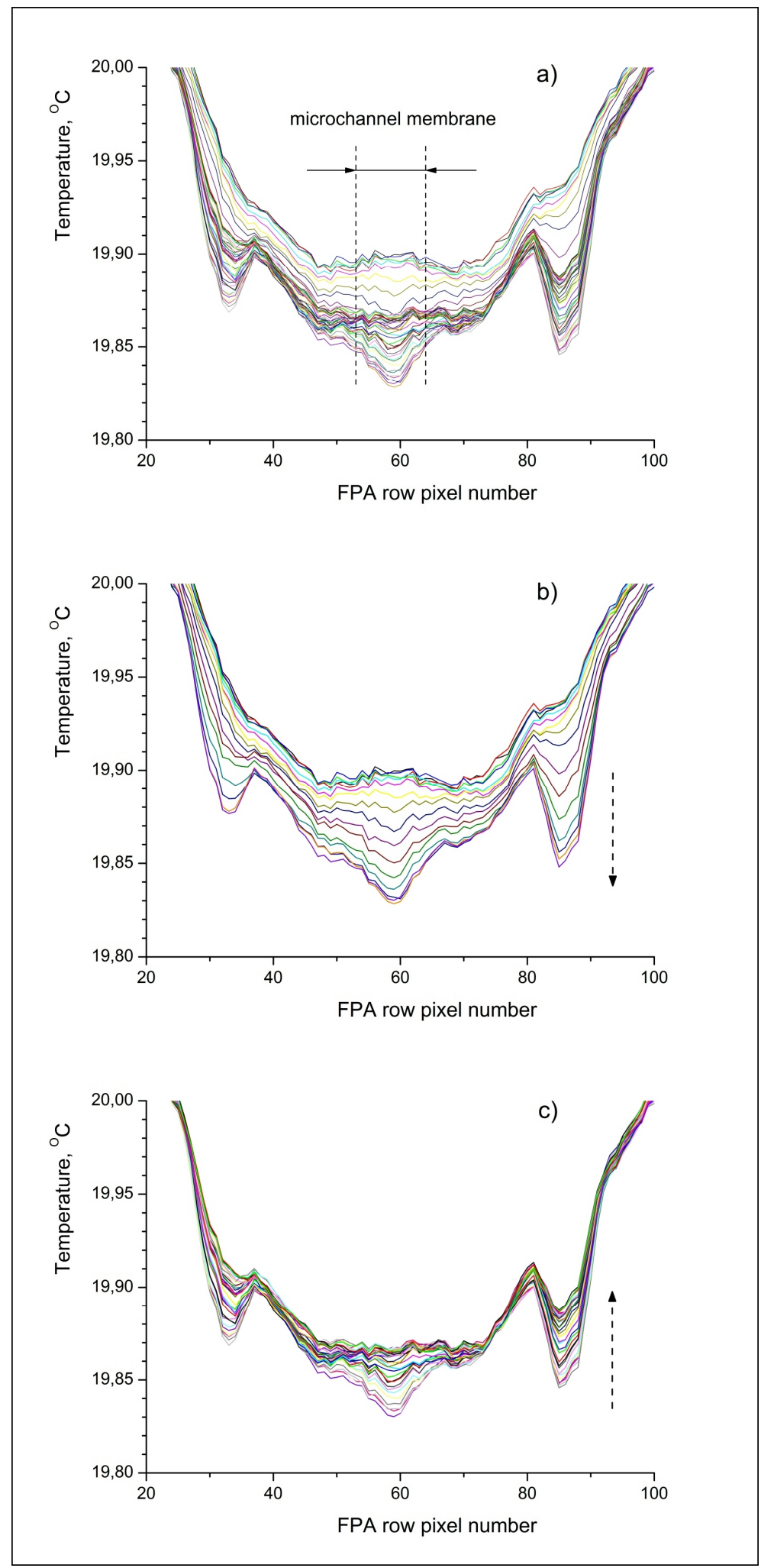

Fig. 6. Quantitative characteristic of water molecules desorption from the container walls and surface of microchannel silicon structure (spacing: $20 \mu \mathrm{m} \times 20 \mu \mathrm{m}$ ). The desorption was resulted from the decrease of surrounding air pressure from $10^{-1}$ to $10^{-3} \mathrm{~atm}$. The temperature profiles were obtained for $9 \mathrm{~s}$ with the interframe intervals of $0.177 \mathrm{~s}$.

a - temperature profiles representing both desorption and the attainment of a quasisteady state, $b$ - desorption stage, $c$ - attainment of a quasisteady state. 


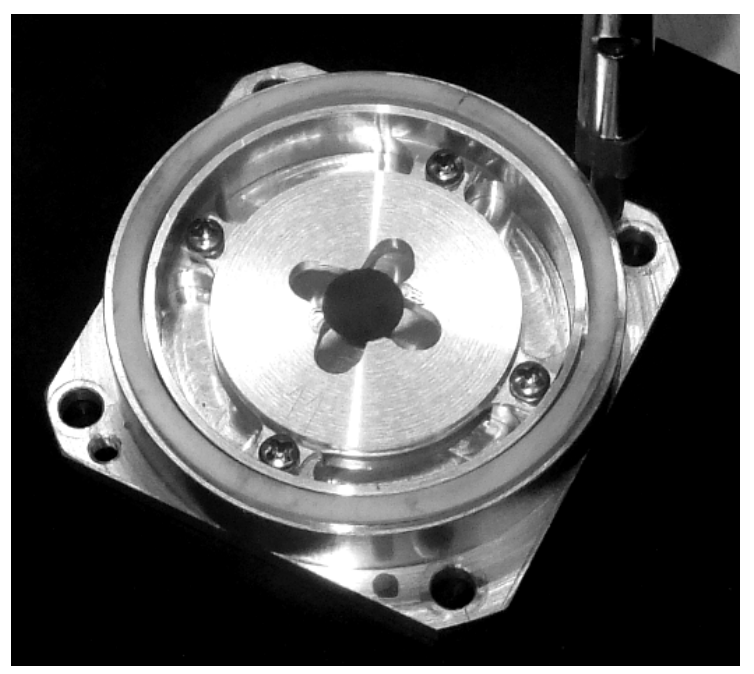

Fig. 7. A hanging microchannel silicon membrane mounted in the container (cell).

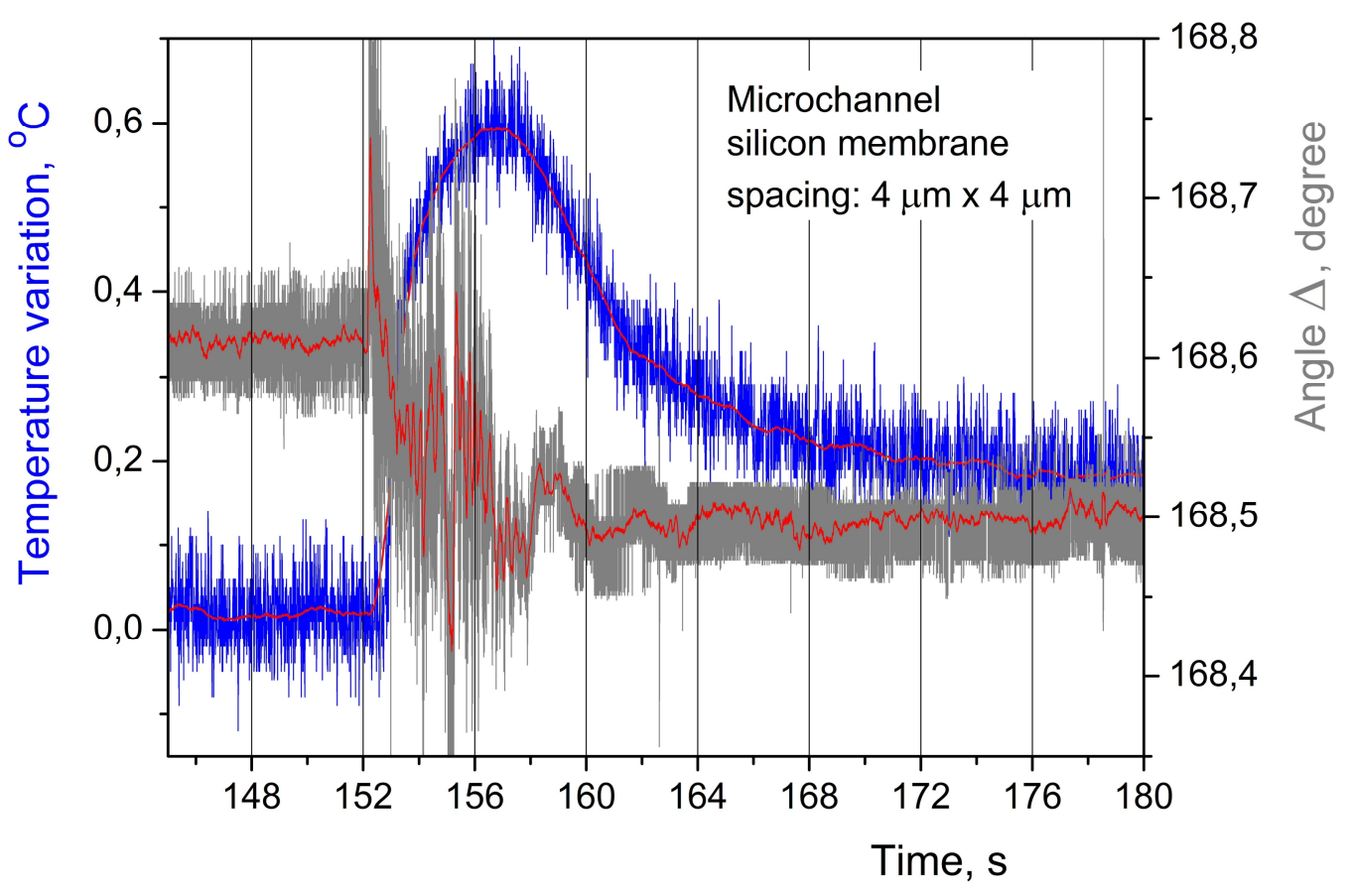

Fig 8. Superimposition of ellipsometric and thermographic representations of the initial stage of atmospheric water molecules adsorption on the surface of a hanging microchannel silicon membrane having the area of $0.5 \mathrm{~cm}^{2}$ and the thickness of $165 \mu \mathrm{m}$. 
http://dx.doi.org/10.21611/qirt.2014.167

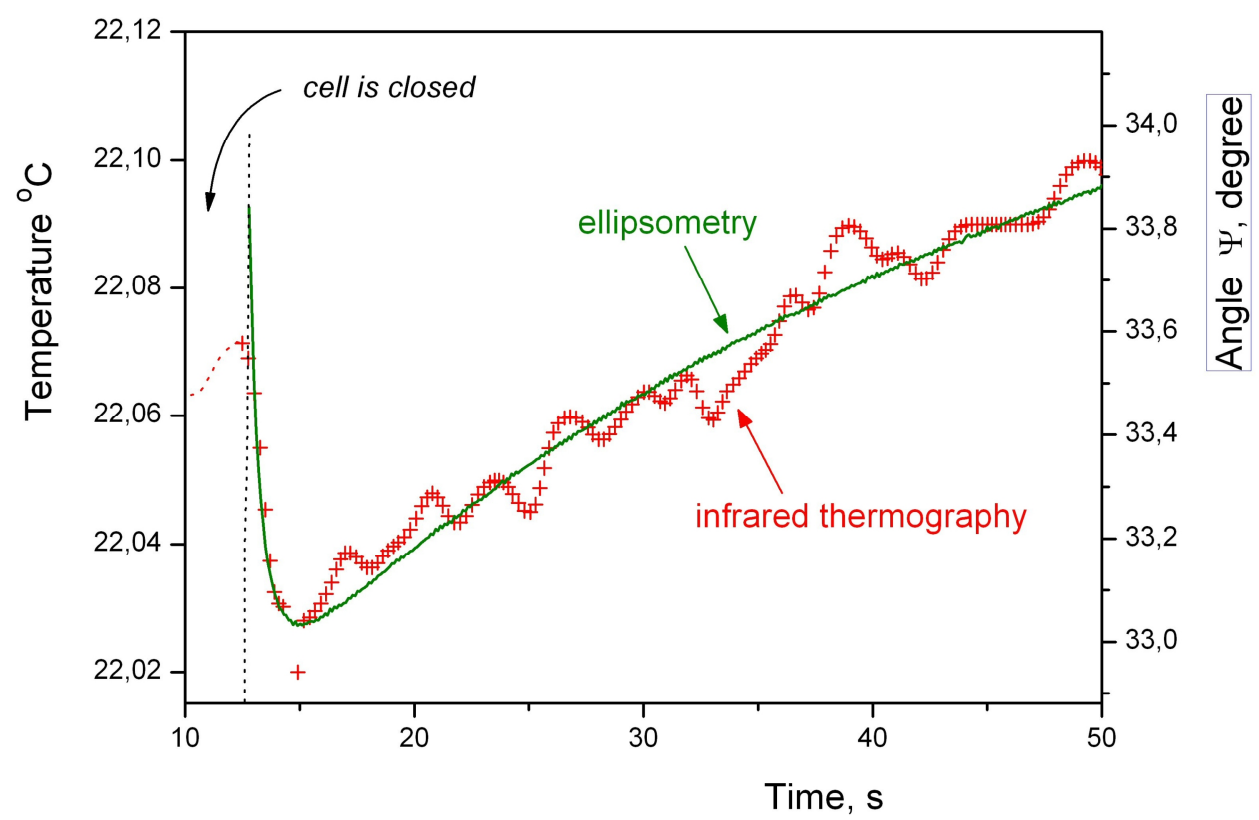

Fig. 9. The initial stage of desorption represented with two high-precision methods - FPA-based infrared thermography and fast laser ellipsometry. 